\title{
Anomalous rotational properties of Bose-Einstein condensates in asymmetric traps
}

\author{
Juan J. García-Ripoll, Víctor M. Pérez-García \\ Departamento de Matemáticas, Escuela Técnica Superior de Ingenieros Industriales, \\ Universidad de Castilla-La Mancha 13071 Ciudad Real, Spain
}

(October 25, 2018)

We study the rotational properties of a Bose-Einstein condensate confined in a rotating harmonic trap for different trap anisotropies. With simple arguments, we obtain the velocity field of the quantum fluid for condensates with or without vortices. While the condensate describes open spiraling trajectories, on the frame of reference of the rotating trap the fluid moves against the trap's rotation. We also find expressions for the angular momentum and linear and Thomas-Fermi solutions for a vortex-less state. In these two limits we find the same analytic relation between the shape of the cloud and the rotation speed. Our predictions are supported by numerical simulations of the mean field Gross-Pitaevskii model.

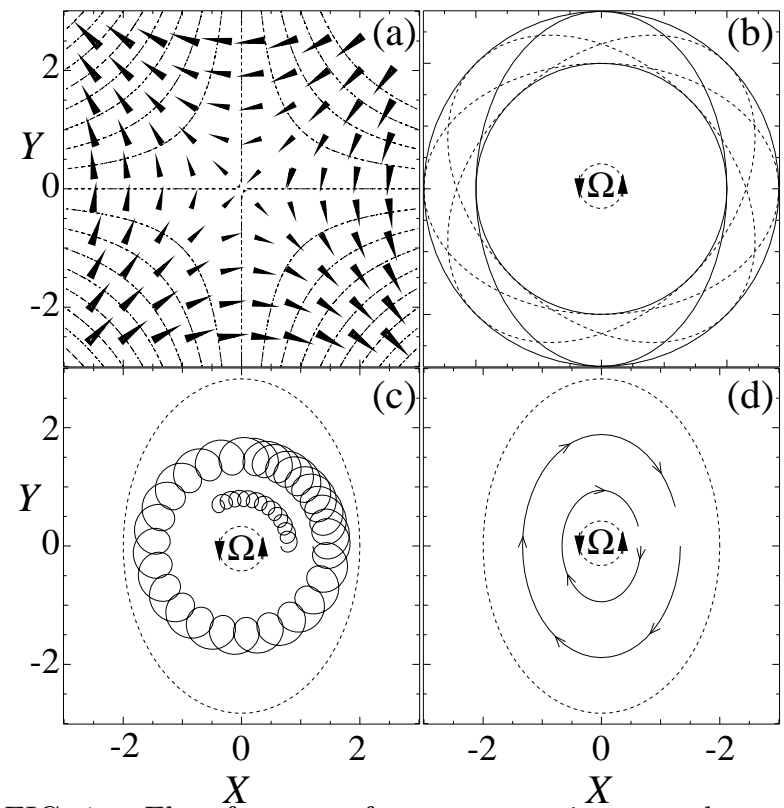

FIG. 1. Flow features of an asymmetric vortex-less condensate with form factor $a \simeq 2$ and rotation speed $\Omega=1$. (a) Velocity distribution at $t=0$ (b) The rotation of the asymmetric condensate defines two circles which define the width of the spiraling flow in the following picture. (c) Flow on the laboratory frame: the shape of the cloud (dashed line) in the Thomas-Fermi limit, and the paths of two test particles - inner spirals for 6 rotation and outer spiral for 20 rotation periods ( 1 period is $2 \pi / \Omega$ ). (d) Flow on the rotating frame using the same case as in (c) but for only one period. The motion of the particles is opposite to the trap rotation.

The question of whether an atomic gas may be a super- fluid and the quest for distinct superfluid properties are among the central goals of recent research with atomic Bose-Einstein condensates (BEC). These targets have been largely inspired by previous achievements on ${ }^{4} \mathrm{He}$ and its superfluid phase He-II, so that from one known property of "classical" superfluids some analog is found for the "new" condensates. To name a few phenomena which have been found this way we can cite the discovery of anomalous sound velocities [1,2], the discovery of a critical velocity for the beginning of viscous damping [3], the study of vortices 44 , 8 and their generation [9,10 and the study of rotational properties such as scissors modes 111,12 or moments of inertia [13.

In this context there has been a great interest in achieving Bose-Einstein condensation with rotating traps, a task which has been completed recently 10,12. The motivation of this interest is that the ground state of a condensate in a rotating trap can be forced to host one or more vortices depending on the angular speed [4, 8], thus providing us with persistent currents which are themselves traces of superfluidity. Although most of the theoretical work regarding the generation and stability of vortices has focused on isotropic traps [5, 8, it is only using truly anisotropic traps that one may expect the condensate to offer a mechanical response to the rotation and produce a vortex. This idea is developed throughout the paper.

There are few relevant results related to non-isotropic traps. First [13], the moment of inertia of an inhomogeneous condensate have been studied assuming that the gas is an ideal one, i. e. without interactions. Among the most striking results is the formula which relates the moment of inertia to the asymmetry of the condensate given by the expectation value $\left\langle x^{2}-y^{2}\right\rangle$. In Ref. [6] the dynamics and stability of a vortex in this type of traps is studied. And finally in Refs. [7] the ground state of a condensate in a rotating asymmetric trap is found numerically for particular values of the rotation speed and their stability is studied.

In this paper we study the rotational properties of a condensate in an rotating anisotropic trap, with and without a vortex. We consider the problem within the mean field approximation, thus taking interactions into account (an essential difference with previous work [13]). Our treatment is both analytic and numeric: we obtain expressions for the most relevant observables, which are exact in some limits, and verify them with complex numerical simulations over a large range of parameters. The 
main predictions of the paper are the explicit expressions for the moments of inertia and a characterization of the flow of the quantum fluid, whose main features are reflected in Fig. 1.

The model.- We will study a two-dimensional condensate in a harmonic trap whose axes rotate at a certain angular speed, $\Omega$. The potential may be described by

$$
V(\mathbf{x}, t)=V_{0}\left(e^{-\Omega t R_{z}} \mathbf{x}\right)=V_{0}(U(-\Omega t) \mathbf{x}),
$$

where $R_{z}$ is the generator of the rotations in the $\Re^{2}$ space, $U(\theta)$ is an orthogonal transformation which rotates a vector an angle $\theta$ around the origin, and $V_{0}\left(r_{1}, r_{2}\right)=$ $\frac{1}{2}\left(\omega_{1}^{2} r_{1}^{2}+\omega_{2}^{2} r_{2}^{2}\right)$.

We will work on two different frames of reference: the laboratory frame $\{S, \mathbf{x}\}$ which is stationary and the rotating frame $\{\tilde{S}, \mathbf{r}=U(-\Omega t) \mathbf{x}\}$ which moves with the trap. On the first frame the zero temperature mean field theory reads

$$
i \partial_{t} \psi(x, t)=\left[-\frac{1}{2} \triangle_{x}+V(x, t)+g|\psi|^{2}\right] \psi(x, t) .
$$

Here $g$ characterizes the interaction and is defined in terms of the ground state scattering length. It is important to remark that our solutions have a well defined norm that we may take as equal to the number of particles 14, $\|\psi\|^{2}=\|\phi\|^{2}=N$. To simplify the treatment we have assumed a set of units in which $\hbar=m=1$.

We will define a second wave function, this time on $\tilde{S}$, given by $\phi(\mathbf{r}, t)=\psi(U(\Omega t) \mathbf{r}, t)$ with $\mathbf{r}=\left(r_{1}, r_{2}\right)$. The evolution of this function is ruled by

$$
i \partial_{t} \phi=\left[-\frac{1}{2} \triangle_{r}+V_{0}(\mathbf{r})+g|\phi|^{2}-\Omega L_{z}\right] \phi .
$$

Here $L_{z}=-i\left(r_{1} \partial_{2} \phi-r_{2} \partial_{1} \phi\right)$ is a representation of the angular momentum operator along the z-axis.

Stationary states.- Let us write Eq. (2) in the modulusphase representation in the rotating frame of reference. Defining $\left(\phi=\sqrt{\rho} e^{i \Theta}\right)$ we obtain the continuity equation for the density

$$
\partial_{t} \rho-\Omega \nabla \rho \cdot\left(R_{z} \mathbf{r}\right)=-\nabla \cdot[\rho \mathbf{v}],
$$

where $\mathbf{v}=\operatorname{Im}(\bar{\phi} \nabla \phi)=\nabla \arg \phi=\nabla \Theta$ is almost the velocity field of the quantum fluid in the stationary frame. Actually the velocity frame on $S$ is given by $\mathbf{V}=U(-\Omega t) \mathbf{v}(U(-\Omega t))$.

Eq. (3) has a set of stationary states, which are solutions of the type $\phi(\mathbf{r}, t)=e^{-i \mu t} \phi(\mathbf{r}, 0)$. These states represent configurations of the condensed cloud which maintain their shape and move rigidly with the trap (keep in mind that $\mathbf{r}$ corresponds to the rotating system).

In our paper we will be interested in the lowest energy state, the ground state. The main assumption throughout the paper will be that the lines of constant density of a ground state are ellipses of an unknown shape, i. e.,

$$
\rho=\rho\left(u^{2}\right)=\rho\left(r_{1}^{2}+r_{2}^{2} / a\right),
$$

which is exact for the ground state in certain limits to be discussed later. We will also need a normalized anisotropy factor, $k=(1-a) /(1+a) \in[-1,+1]$.

Using Eqs (5) and (4), one finds

$$
h(u)\left[\Omega(1-a) r_{1} r_{2}+a r_{1} \partial_{1}+r_{2} \partial_{2}\right] \Theta=\triangle \Theta,
$$

where $h=\mathrm{d} \ln \rho / \mathrm{d}\left(u^{2}\right)$. Let us look for solutions corresponding to an incompressible flow, i.e. $\triangle \Theta=0$. Then

$$
\Omega\left[(1-a) r_{1} r_{2}\right]+\left[a r_{1} \partial_{1}+r_{2} \partial_{2}\right] \Theta=0,
$$

Writing the solution of Eq. (7) in the form

$$
\Theta\left(r_{1}, r_{2}, t\right)=-\mu t+\Omega \frac{a-1}{a+1} r_{1} r_{2}+\Theta_{\text {vort }}\left(r_{1}, r_{2}\right),
$$

it is clear that there is still an undetermined part of the phase, $\Theta_{\text {vort }}$ which satisfies $\triangle \Theta_{\text {vort }}=0$ and $\nabla \Theta_{\text {vort }} \perp$ $\nabla \rho$. This phase varies along elliptic paths surrounding the origin and carries vorticity in case there is any.

Once we have found the phase, the density may be obtained by solving

$$
\mu=-\frac{\triangle \sqrt{\rho}}{2 \sqrt{\rho}}+\frac{(\nabla \Theta)^{2}}{2}+V_{0}+g \rho+i \Omega L_{z} \Theta .
$$

This can be done analytically both in the non-interacting limit, $g N \rightarrow 0$ and on the Thomas-Fermi limit, $g N \rightarrow \infty$ as we will show later.

States without vorticity.- When the BEC ground state has no vorticity we can make $\Theta_{\text {vort }}=0$ in Eq. (8)

$$
\Theta=-\mu t+\Omega \frac{a-1}{a+1} r_{1} r_{2} .
$$

As it was already expected, in the radially symmetric case $(a=0)$ the solution is also radially symmetric, the phase is uniform and the velocity field becomes zero.

For an asymmetric trap the velocity field is different from zero but still irrotacional, $\nabla \times \mathbf{V}=0$. The definition of $\mathbf{V}$ above provides us with analytic expressions for the flow on the laboratory. This flow is a product of two rotations around the origin: a circular rotation with angular speed $\Omega$ and an elliptic rotation with a smaller angular speed $\omega=-\Omega \sqrt{1-k^{2}}$ on the opposite sense! This is a striking two-fold result. First, on the trap frame $\tilde{S}$ the fluid is moving along closed elliptic contours opposite to the rotation of the trap [See Fig. 1(d)] but with a smaller velocity. And second, the composition of both movements on the laboratory gives a flow made of slow and typically open spiraling paths [See Fig. 1(c)].

Roughly speaking, the fact that the actual flow on the laboratory frame is much slower is a signature that the inertia of a condensate is smaller than the inertia of a classical fluid. We will prove this calculating the angular momentum of the cloud, which is given by a simple expression 


$$
\left\langle L_{z}\right\rangle_{\text {ground }}=\int \rho(r \times \nabla \Theta)_{z}=\Omega \frac{\left\langle r_{1}^{2}-r_{2}^{2}\right\rangle^{2}}{\left\langle r_{1}^{2}+r_{2}^{2}\right\rangle}
$$

Here we have used $a=\left\langle r_{1}^{2}\right\rangle /\left\langle r_{2}^{2}\right\rangle$. As it was expected, the moment of inertia, $I=\left\langle L_{z}\right\rangle / \Omega$, becomes zero for a symmetric trap. Furthermore, it is always smaller than the classical value $I=\left\langle r_{1}^{2}+r_{2}^{2}\right\rangle$. We must also remark that that Eq. (11) differs from the zero temperature limit of Ref. 13 because our method is not perturbative and allows the shape of the cloud to depend on the rotation speed.

Inserting Eq. (10) into Eq. (3) we find the equation of a nonlinear harmonic oscillator with a pair of effective frequencies which depend on $\Omega$,

$$
\begin{aligned}
& \omega_{x, \text { eff }}^{2}=\omega_{1}^{2}+\Omega^{2} k(k-2), \\
& \omega_{y, \text { eff }}^{2}=\omega_{2}^{2}+\Omega^{2} k(k+2),
\end{aligned}
$$

We can recover the asymmetry of the cloud from the exact solutions of this oscillator on two different limits, the non-interacting or linear limit, $g \rightarrow 0$, and the ThomasFermi limit. Both expressions are

$$
\begin{aligned}
a_{L} & =a(g \rightarrow 0)=\omega_{x, e f f} / \omega_{y, e f f}, \\
a_{T F} & =a(g \rightarrow \infty)=\left(\omega_{x, e f f} / \omega_{y, e f f}\right)^{2} .
\end{aligned}
$$

It is apparent in Eq. (13) that rotation emphasizes the anisotropy. In Fig. 2(c-d) we compare the Thomas-Fermi and linear approximations with two different numerical experiments with an anisotropic trap. The main conclusion is that Eq. (13(b)) works extremely well for medium to large $N$. There is a small error which depends on the norm of the solution. This error is related to the fact that a Thomas-Fermi approximation wipes out any dependence of the shape of the cloud on the the number of particles, $N$.

Nucleation of the first vortex.- As the rotation speed is increased the fluid adapts by evolving to different states, which may involve the nucleation of one or more vortices. These vortices arise both as zeros of the density and as additive contributions, $\Theta_{\text {vortex }}$, to the phase of the cloud. The phase of the vortices suffers a discontinuous change around any closed circuit which encloses them. Hence vortices satisfy Feynmann's condition for the quantization of the superfluid flow

$$
\oint \nabla \Theta_{\text {vortex }} \cdot \mathrm{d} \mathbf{l}=2 \pi m, \quad m=0, \pm 1, \pm 2 \ldots
$$

Following the reasoning developed throughout this paper, we should now solve a Poisson equation for $\Theta_{\text {vortex }}$ with some restrictions on the direction of $\nabla \Theta_{\text {vortex }}$. Instead we will follow a more intuitive path which gives accurate results. In the framework of our approximation $\Theta_{\text {vortex }}$ will correspond to a flow around the lines of constant density of the cloud, as it happens in the symmetric case. In elliptic polar coordinates, $\left\{r_{1}=u \cos \theta, r_{2}=\sqrt{a} u \sin \theta\right\}$ the gradient of the phase becomes

$$
\nabla \Theta_{\text {vortex }} \simeq g(u)(-\sin \theta, \sqrt{a} \cos \theta),
$$

where $g(u)$ is a decreasing function of the radius of each ellipse. This expression may be integrated along elliptic contours to find the dependence of the vortex phase on the elliptic angle

$$
\Theta_{\text {vortex }}(\theta) \simeq m \theta+m \frac{a-1}{2(a+1)} \sin 2 \theta .
$$

We have found the typical dependence for a symmetric vortex, $m \theta$, plus a correction which comes from the asymmetry. Using Eq. (16) we estimate the new value of the angular momentum. It has two contributions, one coming from the rotation of the cloud and another one coming from the vortex,

$$
\left\langle L_{z}\right\rangle=\Omega \frac{\left\langle r_{1}^{2}-r_{2}^{2}\right\rangle^{2}}{\left\langle r_{1}^{2}+r_{2}^{2}\right\rangle}+\frac{2 m \sqrt{\left\langle r_{1}^{2}\right\rangle\left\langle r_{2}^{2}\right\rangle}}{\left\langle r_{1}^{2}+r_{2}^{2}\right\rangle} N .
$$

In the symmetric trap case the introduction of a vortex implies a fixed change of the total angular momentum $\Delta L_{z}=\left\langle L_{z}\right\rangle_{\text {vortex }}-\left\langle L_{z}\right\rangle_{\text {ground }}=N$. The asymmetry radically changes this picture and the more asymmetric the trap is the smaller the amount angular momentum kept in the vortex - a quantity which eventually becomes zero in the $|k| \rightarrow 1$ limit. (a)
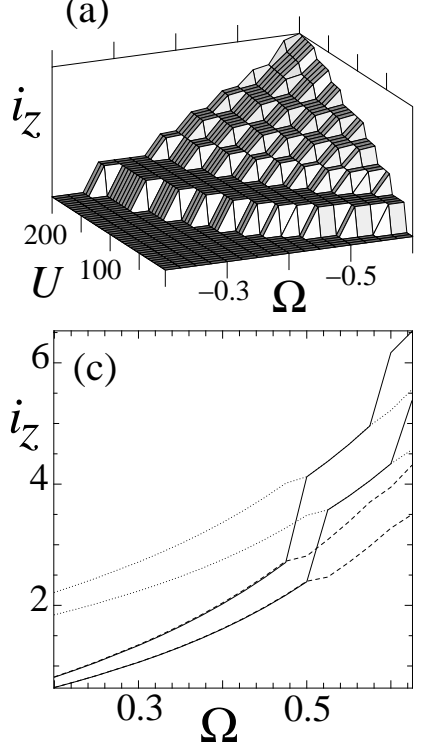
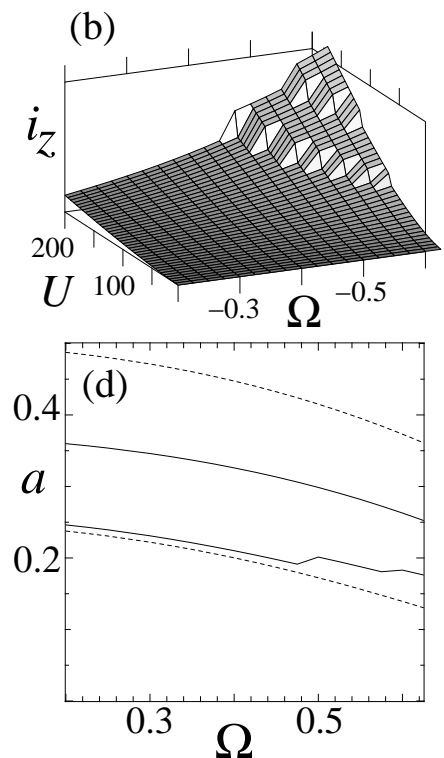

FIG. 2. (a-b) Evolution of the moment of inertia per particle $i_{z}=L_{z} / N \Omega$ as a function of the rotation speed and $N$ for (a) a radially symmetric trap and (b) an asymmetric trap with $\omega_{2}^{2}=2 \omega_{1}^{2}$. (c) Moment of inertia versus the angular speed of the asymmetric trap (solid line), estimation for a vortex-less state (dotted line) and estimation for a state with a single vortex (dashed line). (d) Asymmetry of the cloud, $a$, in the asymmetric trap as theoretical prediction (dotted line), numerical result for $N=5$ (upper solid line) and $N=175$ (lower solid line). All figures are in adimensional units. 
Numerical simulations.- Up to this point we have obtained several predictions concerning the stationary states of a BEC in a rotating trap. Although there are no experimental results on this yet, we can contrast our theoretical work with numerical simulations of the GrossPitaevskii equation (GPE).

First let us recall that the stationary solutions of Eq. (3) are critical points of the functional [8]

$$
E[\phi]=\int \bar{\phi}\left(-\frac{1}{2} \triangle_{r}+V_{0}(r)+\frac{g}{2}|\phi|^{2}-\Omega L_{z}\right) \phi,
$$

subject to the constraint of a fixed norm $\int|\phi|^{2} d^{n} r=N$. Some of the critical points of Eq. (18) correspond to ground states. These states represent the typical configuration of the gas when it is Bose-condensed in the rotating trap. To find the ground states we have minimized Eq. (18) using the Sobolev's gradients method in a discrete Fourier basis. This was performed for a twodimensional radially symmetric trap, $\omega_{1}=\omega_{2}$, and for an asymmetric one, $\omega_{1}=\sqrt{2} \omega_{2}$, varying the only relevant parameters, $\Omega$ and $N$, over a wide range. As a result we obtained two maps which show the angular momentum of the ground state as a function of $(\Omega, N)$, and which are plotted in Fig. 2(a-b).

In Fig. 2(a) we recover the discontinuous distribution which was found in Ref. [4]. Here the system starts with zero angular momentum and remains still until a certain angular speed, $\Omega_{1}(N)$. Beyond $\Omega_{1}(N)$ a vortex grows and we have another plateau on which the angular momentum remains constant, $L_{z}=N$. Once more there is a critical frequency, $\Omega_{2}(N)$, which marks the nucleation of another vortex. From then on the evolution of $L_{z}$ is a piecewise differentiable one. There are still more jumps due to the creation of more vortices, but $L_{z}$ is no longer constant in each interval, since the vortices may move to accommodate more angular momentum.

In Fig. 2(b) the picture is quite different. First the angular momentum has a non-trivial dependence on the number of particles and on the angular speed. It is neither zero for a vortex-less state, nor constant for a state with one vortex. Indeed, the dependence with respect to $\Omega$ is extremely well reproduced by Eq. (11) and by Eq. (17), which suggests that these are exact laws which could be derived by some other mean.

Conclusions.- We have achieved several goals in this work. First, we have shown how to obtain analytic results about the macroscopic properties of a rotating condensate, which leads to unexpected and intuitively appealing results for the flow of the condensate. And second, we have derived precise laws which relate the anisotropy of the condensate, its moment of inertia and the two relevant parameters of the problem: the norm, $N$, and the trap angular speed, $\Omega$. Our predictions have been numerically confirmed generating maps of $L_{z},\left\langle x^{2}\right\rangle$ and $\left\langle y^{2}\right\rangle$ as a function of $(\Omega, N)$.
We hope that these methods will support further work, such as the study of linear stability of vortices in asymmetric condensates, the development of better approximations to the shape of the cloud or even a variational analysis which describes the transition between different vorticities. Furthermore, our predictions are easily extensible to three-dimensional condensates giving many results (condensate shape, moment of inertia or energy release, for instance) which could be tested in current experiments [12]. For instance, the discontinuity on the asymmetry of the cloud when a vortex is nucleated could be used as a non-destructive mean to ensure the existence of a phase singularity in the atomic cloud.

This work has been partially supported by CICYT under grant PB96-0534.

[1] E. Zaremba, Phys. Rev. A 57518 (1998); A. Griffin and E. Zaremba, Phys. Rev. A 564839 (1997).

[2] M. R. Andrews, O. M. Kurn, H.-J. Miesner, D. S. Durfee, C. G. Townsend, S. Inouye and W. Ketterle Phys. Rev. Lett. 79553 (1997).

[3] C. Raman, M. Köhl, R. Onofrio, D. S. Durfee, C. E. Kuklewicz, Z. Hadzibabic and W. Ketterle, Phys. Rev. Lett. 832502 (1999).

[4] D. A. Butts and D. S. Rokhsar, Nature 397 327-9 (1999).

[5] R. J. Dodd et al, Phys. Rev. A 56 (1997) 587; T. Isoshima and K. Machida, Jour. Phys. Soc. Jpn. 66 (1997) 3502; A. A. Svidzinsky and A. L. Fetter, Phys. Rev. A 58 (1998) 3168; A. L. Fetter, J. Low Temp. Phys. 113, 189 (1998); H. Pu et al., 59 (1999) 1533; T. Isoshima and K. Machida, Phys. Rev. A 59 (1999) 2203.

[6] A. A. Svidzinsky and A. L. Fetter, e-print http://xxx.lanl.gov/abs/cond-mat/9811348.

[7] D. L. Feder, C. W. Clark and B. I. Schneider, Phys. Rev. Lett. 824956 (1999); D. L. Feder, C. W. Clark and B. I. Schneider, Phys. Rev. A 61011601 (2000).

[8] J. J. García-Ripoll and V. M. Pérez-García, Phys. Rev. A 60, 4864 (1999).

[9] M. R. Matthews,B. P. Anderson, P. C. Haljan, D. S. Hall, C. E. Wienmann, E. A. Cornell, Phys. Rev. Lett. 832498 (1999).

[10] K. W. Madison, F. Chevy, W. Wohlleben, J. Dalibard, Phys. Rev. Lett. 84806 (2000).

[11] D. Guery-Odelin and S. Stringari, Phys. Rev. Lett. 83 4452 (1999).

[12] O. M. Maragò et al, Phys. Rev. Lett. 842056 (2000).

[13] S. Stringari, Phys. Rev. Lett. 761405 (1996).

[14] For a three-dimensional setup with an almost twodimensional condensate, $N$ is not exactly the number of particles but rather the limit of a three-dimensional one. Several criteria may be used to connect $N$ in the $2 \mathrm{D}$ case to the real number of particles. 